\title{
Spatial Variability Analysis of Farmland Soil Infiltration Based on Model Parameters
}

\author{
Qingdao Xin ${ }^{1}$, Hemin Zhu ${ }^{1}$, Yangren Wang ${ }^{2}$, Xinrui Fan ${ }^{2}$ \\ ${ }^{1}$ Department of Hydraulic Engineering, Tianjin Agricultural University, Tianjin 300384. \\ ${ }^{2}$ University-enterprise collaborative innovation laboratory of water-saving irrigation technology and equipment, Tianjin 300384.
}

\begin{abstract}
Research on the variation of soil infiltration is helpful to analyze the mechanism of soil water movement in farmland. At the same time, soil infiltration characteristics affect the surface irrigation. Based on the field test data, this study simulated and analyzed the soil infiltration with three soil infiltration models (Kostiakov-Lewis model, Philip model and Horton model). The infiltration uncertainty of farmland soil are investigated, and proposed by using two random simulation methods (direct method and parameter mean method) of infiltration. The evaluated indicators are the interval size and its stability of cumulative infiltration amount changed with $95 \%$ confidence. The effects of different random simulations methods and three models on the infiltration process are compared and analyzed. Finally, the model and stochastic simulation method suitable for the infiltration characteristics of the farmland are determined. The results show that the correlation coefficients of the three models are all above 0.98 , and there is no significant difference in fitting accuracy. In terms of the degree of spatial uncertainty (determined by standard deviation): direct method > parameter mean method, in which the combination of the Kostiakov-Lewis model and the parameter mean method have less uncertainty, and the combined simulation effect is better, it is more suitable for the simulation of soil infiltration at farmland scale.
\end{abstract}

\section{Introduction}

Infiltration is one of the important water exchange processes in farmland, and it is also an important factor affecting the uniformity of farmland irrigation ${ }^{[1]}$. Affected by the heterogeneous distribution of soil, infiltration often show strong spatial and temporal variability $^{[23]}$, there are significant variations even on the field scale ${ }^{[4]}$. This causes great difficulty and uncertainty to the precise irrigation of farmland, the parameterization of hydrological models and the simulation of soil hydrological processes ${ }^{[5]}$. Understanding the spatial variability of soil infiltration is of great significance for accurately simulating soil infiltration.

In general, the spatial variability of soil infiltration is more focused on the evaluation of infiltration variability. The research on the effects of spatial variability on the infiltration process is rarely reported. Based on the field soil infiltration test, this study analyzes the soil water infiltration process and its parameter variation and uncertainty on the farmland scale by Kostiakov-lewis, Philip and Horton model. It is hoped that the optimal combination of soil water infiltration model and uncertainty simulation method suitable for the study area will be given, which will provide a theoretical basis for soil infiltration simulation.

\section{Materials and Methods}

\subsection{Test area overview and infiltration test method}

The test area is located in the West Campus of Tianjin agricultural university, Yangliuqing Town, Xiqing District, Tianjin. The test area has 0.9 hectares of arable land, and has good irrigation conditions. Irrigation is generally 2 to 3 times for the wheat growth period, and 1 or 2 times for the corn. The average annual temperature is $12.2^{\circ} \mathrm{C}$ in the region, the average annual rainfall is about $557.3 \mathrm{~mm}$, and the evaporation is $1735.9 \mathrm{~mm}$. The soil texture is medium-light loam. The average bulk weight of soil is $1.42 \mathrm{~g} / \mathrm{cm}^{3}$ in $0-100 \mathrm{~cm}$, the field water holding capacity is $24.0 \%$, and the groundwater depth is 3-5m.

This experiment selects five measuring points from west to east in corn planting farmland $(100 \mathrm{~m} \times 30 \mathrm{~m})$. The distance between each measuring point is $15 \mathrm{~m}$. The first measuring point is $15 \mathrm{~m}$ away from the north and south sides of the corn field, 30 meters from the West side, the fifth point is 10 meters away from the east side, the specific arrangement of the measuring points is shown in Figure 1. The double-ring infiltration instrument (inner ring diameter $30 \mathrm{~cm}$, outer ring diameter $60 \mathrm{~cm}$, height $25 \mathrm{~cm}$ ) was used to measure the infiltration, which was 
divided into four steps: leveling the land (removing surface vegetation and stones, etc.), place the ring (the inner and outer rings are placed vertically in the soil $10 \mathrm{~cm}$ depth, the inner and outer rings are concentric), make the mark (insert the steel ruler on the inner wall of the inner ring, mark $5 \mathrm{~cm}$ away from the soil surface) and add water time (the inner and outer rings maintain the same head $5 \mathrm{~cm}$ ), when the water depth drops by $1 \mathrm{~cm}$, water is added in time and the amount and time of the added water is recorded. When the water addition interval is close, the test can be ended.

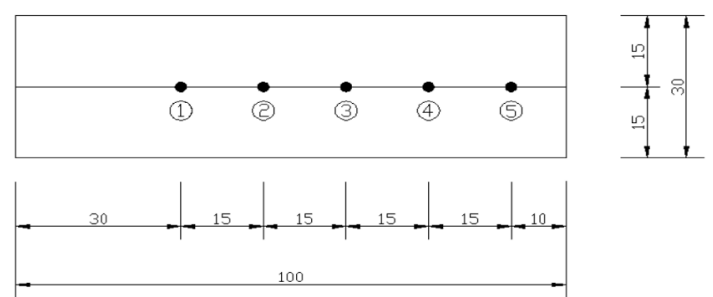

Figure 1 Layout of the infiltration test point (unit: m)

\subsection{Stochastic simulation based on spatial variability of cumulative infiltration.}

The spatial variation of soil infiltration in farmland means that the infiltration characteristics are random with the change of spatial point, that is, the amount of water infiltrated at different spatial points in the same infiltration time is different, and the infiltration rate is also different. The main reason for the uncertainty is the difference in soil texture, profile distribution structure and soil moisture content before infiltration at different spatial points. The spatial uncertainty of soil infiltration in farmland can be expressed by the mean value and standard deviation of the soil infiltration data. The value can be determined by analyzing the soil infiltration test data at different spatial points in the farmland.

Objectively, the spatial uncertainty of soil infiltration is certain. However, different analytical methods, different field infiltration test points will yield different variability. This paper selects Kostiakov-Lewis (hereafter abbreviated as K-L), Horton model and Philip model (see Table 1), analyzes the spatial variability of the model parameters, and based on this, simulates the spatial variability of farmland soil infiltration, and simulation of the spatial uncertainty of the infiltration is carried out based on the spatial uncertainty of accumulation infiltration, targeting the uncertainty interval as small and stable as possible, a method for expressing the spatial uncertainty of soil infiltration is given.

Table 1 Introduction of infiltration model

\begin{tabular}{ccc}
\hline Model name & $\begin{array}{c}\text { Cumulative infiltration } \\
\text { amount } / \mathrm{mm}\end{array}$ & Model parameter \\
\hline Philip & $I=S t^{\frac{1}{2}}+A t$ & $S 、 A$ \\
K-L & $I=K t^{\alpha}+f_{0} t$ & $K 、 \alpha 、 f_{0}$
\end{tabular}

$$
\text { Horton } \quad I=i_{c} t+\frac{1}{\beta}\left(i_{0}-i_{c}\right)\left(1-\quad i_{c} 、 i_{0}, \beta\right.
$$

Notes: $I(t)$ is the cumulative infiltration, $\mathrm{cm} ; i_{c}$ is the stable infiltration rate; $i_{0}$ is the initial infiltration rate; $\beta$ is the empirical constant related to soil properties; $t$ is the infiltration time , min. $S$ is the soil moisture absorption rate; $A$ is the stable infiltration rate; $K$ is infiltration coefficient; $\alpha$ is infiltration attenuation rate; $f_{0}$ is stable infiltration rate, $\mathrm{cm} / \mathrm{min}$.

The stochastic simulation based on the spatial variability of cumulative infiltration is carried out by using the average value (equation (1)), the standard deviation (formula (2)) and the deviation coefficient are analyzed (equation (3)), the method is referred to herein as the direct method. Greater the standard deviation and the deviation coefficient, larger is the uncertainty of the infiltration; on the contrary, less uncertain of the infiltration of farmland soils.

$$
\bar{I}(t)=\frac{1}{n} \sum_{j=1}^{n} I_{j}(t)
$$

$$
\begin{gathered}
\sigma_{I}(t)=\sqrt{\frac{1}{n-1} \sum_{j=1}^{n}\left[I_{j}(t)-\bar{I}(t)\right]^{2}} \\
C_{v I}(t)=\frac{\sigma_{I}(t)}{\bar{I}(t)}
\end{gathered}
$$

Where, $\left(\bar{I}_{j}(t)\right)$ is the cumulative infiltration amount tested at the $\mathrm{j}$-th point at the time $t$, min, which is the average value of the accumulated infiltration amount $\left(\bar{I}_{j}(t)\right)$ at all measuring points, $t ; j$ is the number of measuring points in the infiltration test, $j=1,2, \ldots, n, n$ is the number of points.

The stochastic simulation of cumulative infiltration based on the spatial variation of cumulative infiltration can be simulated by equation (4) ${ }^{[6]}$,

$$
\hat{I}(t)=\bar{I}(t)+\varepsilon \sigma_{I}(t)
$$

Where, $\varepsilon$ is a random sequence, which follows the normal distribution $\mathrm{N}(0,1)$, and the other symbols have the same meaning as before. Wherein, the normal random sequence $\varepsilon$ is generated by using a transformation method, such as equation (5),

$$
\left\{\begin{array}{l}
\varepsilon_{1}=\sqrt{-2 \ln u_{1}} \cos 2 \pi u_{2} \\
\varepsilon_{2}=\sqrt{-2 \ln u_{1}} \sin 2 \pi u_{2}
\end{array}\right.
$$

Where $\mathrm{u}_{1}$ and $\mathrm{u}_{2}$ are uniform random numbers over the interval $[0,1]$; and are purely random sequences of standard normal distribution independent of each other, $\varepsilon \sim N(0,1)$.

\subsection{Stochastic simulation based on spatial variability of parameters}

In order to determine the infiltration law of farmland soil, $\mathrm{M}$ spatial points are selected to measure the infiltration data, and the $\mathrm{M}$ group model parameters can be obtained. The mean and standard deviation of the corresponding model are obtained, and the random simulation is carried out. This is the parameter mean method. The stochastic 
simulation corresponding to the $\mathrm{K}-\mathrm{L}$ model is simulated as follows.

$$
K_{s}=\overline{K_{s}}+\sigma_{K_{s}} \cdot \varepsilon_{K_{s}}, \quad \alpha=\bar{\alpha}+\sigma_{\alpha} \cdot \varepsilon_{\alpha}, f_{0}=\overline{f_{o}}+\sigma_{f_{0}} \cdot \varepsilon_{f_{0}}
$$

The simulation method for each parameter corresponding to the Horton model is as follows.

$i_{c}=\overline{i_{c}}+\sigma_{i_{c}} \cdot \varepsilon_{i_{c}}, i_{0}=\overline{i_{0}}+\sigma_{i_{0}} \cdot \varepsilon_{i_{0}}, \beta=\bar{\beta}+\sigma_{\beta} \cdot \varepsilon_{\beta}$

The simulation method for each parameter corresponding to the Philip model is as follows.

$$
S=\bar{S}+\sigma_{S} \cdot \varepsilon_{S}, A=\bar{A}+\sigma_{A} \cdot \varepsilon_{A}
$$

Where $\overline{\mathrm{K}_{\mathrm{s}}}, \bar{\alpha}, \overline{\mathrm{f}_{\mathrm{o}}}, \overline{\mathrm{i}_{\mathrm{c}}}, \overline{\mathrm{i}_{0}}, \bar{\beta}, \overline{\mathrm{S}}$ and $\overline{\mathrm{A}}$ are the average values of the corresponding parameters of the $M$ group infiltration test; respectively $\varepsilon_{\mathrm{K}_{\mathrm{s}}}, \varepsilon_{\alpha}, \varepsilon_{f o}, \varepsilon_{\mathrm{i}_{\mathrm{c}}}, \varepsilon_{\mathrm{i}_{0}}, \varepsilon_{\beta}$, $\varepsilon_{\mathrm{S}}$ and $\varepsilon_{\mathrm{A}}$ are the random sequences of the corresponding parameters obeying the normal distribution $\mathrm{N}(0,1) ; \sigma_{K_{s}}, \sigma_{\alpha}, \sigma_{f_{0}}, \sigma_{i_{c}}, \sigma_{i_{0}}, \sigma_{\beta}$, $\sigma_{S}$ and $\sigma_{A}$ are the standard deviation of the parameters, which are functions of the infiltration time. Other symbols have the same meaning as before.

The uncertainty of the cumulative infiltration in the parameter mean method is expressed by the cumulative infiltration amount change interval obtained by $95 \%$ confidence. In order to avoid unreasonable data during the random simulation of the model parameters, the parameter values determined based on the measured data are used to obtain the variation interval according to the $95 \%$ confidence (see equation (9)). The method uses the uncertainty of the infiltration model parameters to describe the spatial variability of infiltration and is more explanatory to the soil infiltration process.

$$
y=\bar{y} \pm \frac{\sigma}{\sqrt{n}} \cdot \mathrm{Z}_{\alpha / 2}
$$

Where, $y$ is the upper and lower confidence limit of the parameter; $\bar{y}$ is the average of the parameters; $\sigma$ is the standard deviation; $\mathrm{n}$ is the number of measuring points; $a$ is the confidence level; $Z_{\alpha / 2}$ it is found by the normal distribution table.

\section{Results analysis}

\subsection{Analysis of parameter fitting results}

The soil infiltration data of five measuring points were analyzed, and the three infiltration models (Table 1) were fitted and analyzed, and the model parameters fitting

\begin{tabular}{|c|c|c|c|c|c|c|c|c|c|}
\hline \multirow{2}{*}{ model } & \multirow{2}{*}{ parameter } & \multicolumn{5}{|c|}{ Measured point } & \multirow{2}{*}{ average } & \multirow[b]{2}{*}{$\sigma$} & \multirow{2}{*}{$\mathrm{C}_{\mathrm{V}} / \%$} \\
\hline & & 1 & 2 & 3 & 4 & 5 & & & \\
\hline \multirow[t]{4}{*}{ K-L } & $\mathrm{k}$ & 17.813 & 15.683 & 22.799 & 19.475 & 16.198 & 18.3936 & 2.8759 & 15.63 \\
\hline & $f_{0}$ & 0.2918 & 0.3865 & 0.4742 & 0.5023 & 0.2533 & 0.38162 & 0.1092 & 28.61 \\
\hline & $\mathrm{a}$ & 0.2829 & 0.2611 & 0.2464 & 0.2800 & 0.4039 & 0.2949 & 0.0627 & 21.28 \\
\hline & $\mathrm{R}^{2}$ & 0.9974 & 0.9946 & 0.9931 & 0.9982 & 0.9967 & 0.9960 & & \\
\hline \multirow[t]{4}{*}{ Horton } & $i_{c}$ & 0.5677 & 0.5975 & 0.7219 & 0.7775 & 0.8052 & 0.6940 & 0.1065 & 15.35 \\
\hline & $i_{0}$ & 15.7018 & 12.8365 & 18.1301 & 13.7633 & 11.5290 & 14.3921 & 2.5836 & 17.95 \\
\hline & $\beta$ & 0.4058 & 0.3901 & 0.3869 & 0.3058 & 0.2267 & 0.3431 & 0.0758 & 22.10 \\
\hline & $\mathrm{R}^{2}$ & 0.9960 & 0.9992 & 0.9992 & 0.9976 & 0.9948 & 0.9974 & & \\
\hline \multirow[t]{2}{*}{ Philip } & $S$ & 9.672 & 9.117 & 12.026 & 12.086 & 13.007 & 11.1816 & 1.6885 & 15.10 \\
\hline & $\begin{array}{c}A \\
\mathrm{R}^{2}\end{array}$ & 0.9781 & 0.9845 & 0.9784 & 0.9895 & 0.9946 & 0.9850 & & \\
\hline
\end{tabular}
results were obtained (see Table 2).

Table 2 Infiltration model parameter fitting results

From the model fitting results, the average relative error of the simulated of the Horton model is the smallest (3.1\%), and the average relative error of the Philip model is the largest $(8.7 \%)$; the $\mathrm{Cv}$ of each model parameter is between $0.153 \sim 0.286$, the standard deviation is between $0.062 \sim 2.876$. The correlation coefficient of Horton model is the largest, reaching 0.9974, and the Philip model is smaller, but the total is above 0.98 , which indicates that the simulation accuracy of each model is high for farmland infiltration.

\subsection{Spatial uncertainty analysis of infiltration}

The spatial uncertainty analysis of soil infiltration aims to give the optimal combination of model and stochastic simulation method to describe the uncertainty of farmland soil infiltration. The analysis is based on different stochastic simulation methods as follows.

Using the data of five test points, according to formula (2), the standard deviation of the infiltration is obtained. The random simulation of cumulative infiltration is performed by equation (4), and the number of simulations is 200 times. The confidence upper and lower limits of the cumulative infiltration simulation at $95 \%$ confidence based on table 3 and the mean and standard deviation (equation (1) and (2)) are shown in Figure 2. In order to illustrate the stability of the upper and lower confidence simulation results, three sets of random simulations were performed. Each group was carried out to 200 random simulations. Three infiltration times were selected for 30,90 and 130 minutes, and the corresponding time cumulative infiltration amount was given. The lower and upper limit and its $\mathrm{Cv}$ are shown in Table 4. 
Table 3 Confidence upper and lower limits of parameter random simulation

\begin{tabular}{cccccccc}
\hline Model & \multicolumn{3}{c}{$\mathrm{K}-\mathrm{L}$} & & Horton & \multicolumn{2}{c}{ Philip } \\
\hline parameter & $K$ & $f_{o} / \mathrm{mm} / \mathrm{min}$ & $\alpha$ & $i_{o} / \mathrm{mm} / \mathrm{min}$ & $i_{c} / \mathrm{mm} / \mathrm{min}$ & $\beta$ & $S$ \\
\hline upper limit & 23.2 & 0.6 & 0.4 & 16.7 & 0.8 & 0.4 & 12.7 \\
Lower limit & 11.4 & 0.1 & 0.2 & 12.1 & 0.6 & 0.3 & 9.7 \\
\hline
\end{tabular}

The results (Figure 2) show that the cumulative infiltration of the five points is within the upper and lower confidence limits; the upper confidence limit increases with the infiltration time, and the lower confidence limit increases too slowly with the infiltration time, among them. The simulated results based on K-L model parameters are the best, and the simulation results are more stable (Table 4).

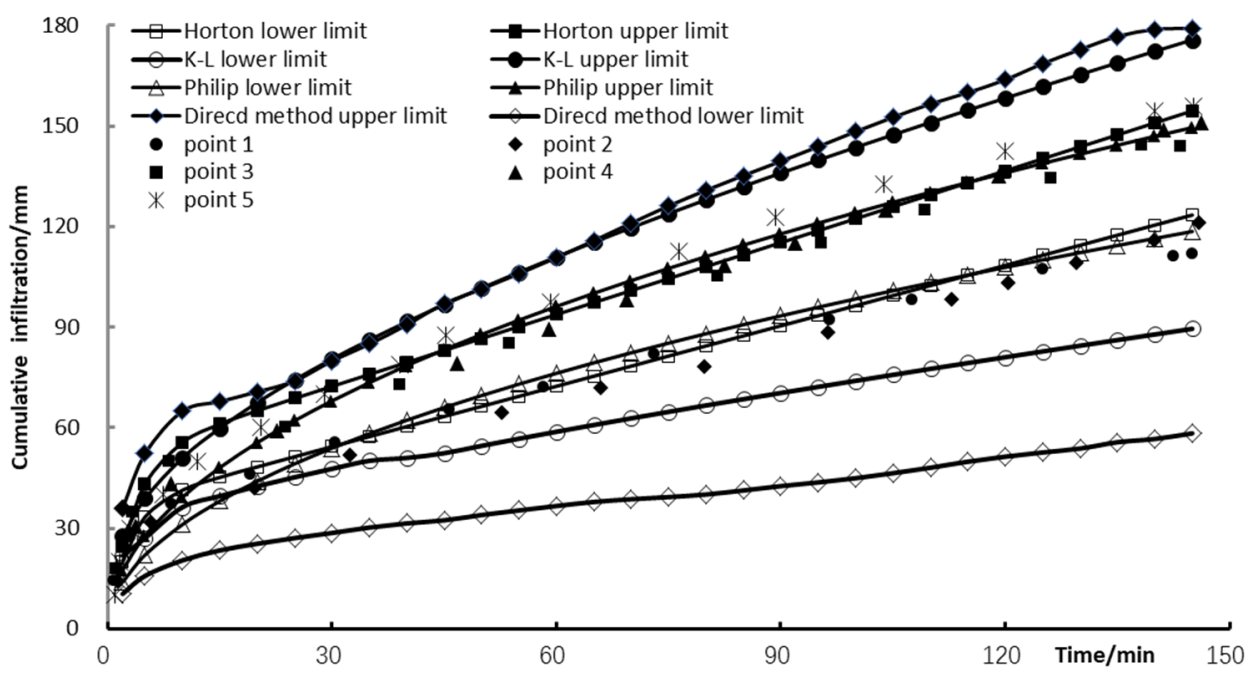

Figure 2 The confidence upper and lower limits based on model parameters and direct method stochastic simulation

Table 4 Confidence upper and lower limit for cumulative infiltration

\begin{tabular}{|c|c|c|c|c|c|c|c|c|c|c|}
\hline \multirow{3}{*}{$\begin{array}{l}\text { simulation } \\
\text { method }\end{array}$} & \multirow{3}{*}{ model } & \multirow{3}{*}{$\begin{array}{l}\text { time / } \\
\text { min }\end{array}$} & \multicolumn{8}{|c|}{ Number of replicates randomly simulated (200 times/group) } \\
\hline & & & \multicolumn{2}{|r|}{1} & \multicolumn{2}{|c|}{2} & \multicolumn{2}{|c|}{3} & \multicolumn{2}{|c|}{$\mathrm{C}_{\mathrm{V}} / \%$} \\
\hline & & & $\begin{array}{c}\text { upper } \\
\text { limit }\end{array}$ & $\begin{array}{l}\text { Lower } \\
\text { limit }\end{array}$ & $\begin{array}{l}\text { upper } \\
\text { limit }\end{array}$ & $\begin{array}{l}\text { Lower } \\
\text { limit }\end{array}$ & $\begin{array}{l}\text { upper } \\
\text { limit }\end{array}$ & $\begin{array}{c}\text { Lower } \\
\text { limit }\end{array}$ & $\begin{array}{l}\text { upper } \\
\text { limit }\end{array}$ & $\begin{array}{c}\text { Lower } \\
\text { limit }\end{array}$ \\
\hline \multirow{3}{*}{ Direct } & \multirow{3}{*}{-— } & 30 & 73.7 & 45.7 & 73.7 & 48.4 & 74.9 & 48.2 & 0.8 & 2.6 \\
\hline & & 90 & 128.5 & 73.9 & 128.5 & 79.1 & 130.8 & 78.7 & 0.8 & 3.1 \\
\hline & & 130 & 158.3 & 94 & 158.3 & 100.1 & 161 & 99.7 & 0.8 & 2.8 \\
\hline \multirow{3}{*}{$\begin{array}{l}\text { Parameter } \\
\text { mean }\end{array}$} & \multirow{3}{*}{ K-L } & 30 & 80.8 & 45.2 & 82 & 45.2 & 78.8 & 45.8 & 1.6 & 0.6 \\
\hline & & 90 & 136 & 70.4 & 140.3 & 72.5 & 135.2 & 72.7 & 1.6 & 1.4 \\
\hline & & 130 & 166.3 & 84.9 & 172.9 & 86.4 & 166.8 & 85.7 & 1.8 & 0.7 \\
\hline
\end{tabular}

\section{Conclusion}

(1) For the soil infiltration of farmland, considering the parameter structure of the model and the Spatial uncertainty of infiltration based on the measured data, two stochastic simulation methods for the farmland infiltration are proposed, namely the direct method and the mean method of the parameters. The parameter mean method based on the K-L model is better.

(2) In terms of the size of the uncertainty interval and the stability of the upper and lower limits of the interval, the K-L model has the best combination simulation effect and can be used to simulate the soil infiltration on the farmland scale.

\section{Acknowledgments.}

The work is supported by the National Natural Science Foundation of China ( 51779174 ), the Tianjin University Students Innovation and the Entrepreneurship Project (201810061024) and Tianjin Agricultural Science and Technology Achievements Transformation and Promotion Project (201701150).

\section{References}

1. He D.Spatial variability of soil infiltration characteristics and its variation sources. Advances in Water Science, 2013, 24(3): 340-348. 
2. Jaynes D B, Clemmens A J. Accounting for spatially variable infiltration in border irrigation models[ J] .Water Resour .Res., 1986, 22(8):1257-1262.

3. Jaynes D B, Hunsaker D J. Spatial and temporal variability of water content and infiltration on a flood irrigated field[ J] .Trans ASAE,1989, $32: 1229-1238$.

4. Achouri M, Gifford G F. Spatial and seasonal variability of field measured infiltration rates on a rangeland site inutah[ J] .J.Range Manage,1984, 37(5):451-455 .

5. Keur Pvander, Iversen BV. Uncertainty in soil physical data at river basin scale: A review [J] . Hydrology and Earth System Sciences,2006,10( 6):889-902.

6. Wang W S, Jin J L, Din J. Random Hydrology [M]. Beijing: China Water Resources and Hydropower Press, 2016. 